\title{
Immunohistochemistry as a tool for screening rare renal cancers
}

\author{
Cissy Yong ${ }^{1,2}$, Grant D. Stewart ${ }^{1,2}$, Christian Frezza ${ }^{3}$ \\ ${ }^{1}$ Department of Surgery, University of Cambridge, Cambridge Biomedical Campus, Cambridge, UK; ${ }^{2}$ Cambridge University Hospitals NHS \\ Foundation Trust, Cambridge, UK; ${ }^{3}$ Medical Research Council Cancer Unit, University of Cambridge, Cambridge Biomedical Campus, \\ Cambridge, UK \\ Correspondence to: Christian Frezza. Medical Research Council Cancer Unit, University of Cambridge, Cambridge Biomedical Campus, CB2 0XZ, \\ UK. Email: cf366@mrc-cu.cam.ac.uk; Grant D. Stewart. Department of Surgery, University of Cambridge, Cambridge Biomedical Campus, CB2 \\ 0QQ, UK; Cambridge University Hospitals NHS Foundation Trust, Cambridge CB2 0QQ, UK. Email: gds35@cam.ac.uk. \\ Provenance: This is an invited article commissioned by the Section Editor Dr. Xiao Li (Department of Urology, Jiangsu Cancer Hospital, Jiangsu \\ Institute of Cancer Research, Nanjing Medical University Affiliated Cancer Hospital, Nanjing, China). \\ Comment on: Gupta S, Swanson AA, Chen YB, et al. Incidence of succinate dehydrogenase and fumarate hydratase-deficient renal cell carcinoma \\ based on immunohistochemical screening with SDHA/SDHB and FH/2SC. Hum Pathol 2019;91:114-22.
}

Submitted Sep 23, 2019. Accepted for publication Sep 30, 2019.

doi: $10.21037 / \mathrm{atm} .2019 .10 .18$

View this article at: http://dx.doi.org/10.21037/atm.2019.10.18

Recent additions of succinate dehydrogenase (SDH) and hereditary leiomyomatosis and renal cell cancer (HLRCC, associated) fumarate hydratase (FH)-deficient renal cell carcinomas (RCC) to the 2016 WHO Classification of Renal Tumours (1) have highlighted an evolving need for the distinction between renal cancer subtypes. Differences in molecular characterisation, clinical phenotypes, and therapeutic responses (1-3) further corroborates this paradigm shift and strongly points towards the development of subtype-specific management (3). SDH and $\mathrm{FH}-$ deficient RCCs are rare tumours strongly associated with hereditary neoplastic syndromes and early-onset $(2,4,5)$. FH-deficient RCCs are highly aggressive tumours associated with poor patient prognosis $(2,3,6)$, whereas SDH-deficient RCCs are phenotypically more variable, but also known to exhibit aggressive disease behaviour (7-9). Whilst localised RCCs (regardless of subtype) are usually managed surgically $(5,7,10)$, in advanced/ metastatic RCCs where systemic therapies are the mainstay of management $(7,10)$, the majority of clinical trials that provide the basis for these guidelines are centred around clear cell RCC (11). Limited and/or no data is available to guide management of metastatic SDH-deficient RCC and, in particular, FH-deficient RCC $(5,7)$. Furthermore, there is a lack of consensus as to the follow-up regime of patients with resected sporadic RCCs, and little evidence on how to observe SDH- or FH-deficient RCCs (7).
Therefore, logic prevails on the strong emphasis placed on improving the accurate detection and diagnosis of these rare subtypes $(9,12-16)$ to discern the phenotypic features more comprehensively and improve patient stratification in the evolving era of precision-based medicine.

Inactivating mutations rendering $\mathrm{SDH}$ and $\mathrm{FH}$ enzyme activity absent lead to the accumulation of succinate and fumarate respectively, which are bona fide oncometabolites that have pro-oncogenic capabilities such as the induction of protein modifications, aggressive tumour phenotypes, and epigenetic modulation $(17,18)$. In particular, fumarate accumulation was shown to bind reactive thiol residues of proteins inducing a post-translational modification called succination $(15,19)$. Of note, succinated proteins can be detected using antibodies against succinated cysteine residues (anti-2SC). In the manuscript recently published in Human Pathology, Gupta and colleagues (20) capitalise on this unique oncometabolite-associated trait and used immunohistochemistry (IHC) to probe SDHA/SDHB and FH/2SC for the detection of SDH and FH-deficient RCCs in a large historical panel of resected renal tumour subtypes $(\mathrm{n}=1,009)$.

The authors used qualitative scoring [i.e. positive $(+)$ or negative (-) comparative to an internal positive control] for SDHA, SDHB, and FH staining. Positive 2SC (2SC+) staining was determined based on a previous study that correlated an intensity rating and staining pattern of 2SC 
with molecular analyses of $F H$ mutations in RCC tissues (12). Tissue sections with abnormal IHC staining underwent pathological review using the WHO 2016 classification and cases were clinically correlated with patient records. Overall, three cases of SDH-deficient RCCs (SDHA+/SDHB-) were identified, and were all tumours originally diagnosed as oncocytomas $(1.1 \%, \mathrm{n}=273)$. Retrospective review of these tumours identified cardinal histological features in keeping with SDH-deficient RCC. Clinically, these patients presented with localised tumours (stage pT2 or lower) with no disease recurrence or adverse outcomes reported on follow up. Four cases of FH-deficient RCC were identified in a subset of tumours stained for $\mathrm{FH} / 2 \mathrm{SC}(\mathrm{n}=730)$. Two cases were identified in the papillary RCC cohort $(0.5 \%$, $\mathrm{n}=400$ ), and 2 cases in the unclassified RCC cohort (4.4\%, $\mathrm{n}=46$ ). Characteristic absence of $\mathrm{FH}$ staining coupled with positive 2SC staining (FH-/2SC+) was reported in 3 cases, whereas one case (papillary RCC) exhibited retained $\mathrm{FH}$ expression with positive $2 \mathrm{SC}$ staining $(\mathrm{FH}+/ 2 \mathrm{SC}+)$. These tumours exhibited a highly aggressive phenotype with three patients developing metastatic disease, and in all four cases proved fatal. Unfortunately, confirmatory $\mathrm{SDH} / \mathrm{FH}$ genetic analyses were not provided in this study.

This study reiterates the rarity of SDH- and FH-deficient RCC subtypes, and underlines some of the important caveats related to aberrant/indeterminate IHC staining and morphological heterogeneity of these rare subtypes. Although all 3 cases of SDH-deficient RCCs were detected in the oncocytoma cohort, the original diagnosis of these cases occurred between 1970 to 2012, before the addition of SDH-deficient RCC to the WHO 2016 Classification (1). It is likely that as awareness of these rare subtypes gains traction, the identification of the associated cardinal morphological features will lead to increased detection and diagnosis, which would be in keeping with this study's pathological review reporting classic morphology consistent with SDH-deficient RCC in all 3 cases. Although it is possible that detecting classic morphological SDH-deficient RCC may be sufficient without IHC, the presence of variant histology has been previously reported for SDH-deficient RCC $(8,21)$. In addition, this study identified 2 cases of FH-deficient RCCs of unclassified morphology. Both cases had highly aggressive disease behaviour in keeping with FH-deficient RCC. One patient had early onset of disease (22 years old) and in both cases, presented with locally advanced disease with subsequent metastatic spread, suggesting the clinical phenotype may remain consistent despite variant histology.
A limitation to this study is the lack of confirmatory molecular analyses to substantiate the authors' claims. In the case of aberrant $\mathrm{FH}+/ 2 \mathrm{SC}+$ staining, the authors suggested the possibility of dysfunctional $\mathrm{FH}$ protein in FH-deficient RCC, accounting for the retained $\mathrm{FH}$ expression on IHC. This hypothesis was backed by the authors reporting a separate case of a HLRCC patient harbouring a $\mathrm{FH}$ germline mutation exhibiting $\mathrm{FH}+2 \mathrm{SC}+$ staining on IHC. However, previous similar studies coupled with molecular analyses were able to identify cases of aberrant $\mathrm{FH}+/ 2 \mathrm{SC}+$ staining with wildtype $F H$ expression, as well as with $F H$ mutations $(14,22)$. We agree with the authors in that concurrent use of $2 \mathrm{SC}$ with $\mathrm{FH}$ staining is essential for the detection of FH-deficient RCCs, furthermore, aberrant staining patterns crucially need to be validated with $F H$ molecular analysis before confirmation of disease. Of note, degradation of tissue specimens in this study posed a major issue in performing crucial molecular analyses and highlights a need for better technical methods in ensuring suitable sample preservation for multiple lines of testing. Other metabolic markers that exploit these unique oncometabolite-associated properties also show promise in detecting FH-deficient RCC. In pre-clinical models of $\mathrm{FH}$ loss, consistently elevated levels of urinary argininosuccinate as a result of fumarate-induced urea cycle metabolic reprogramming (23), as well as observed cellular accumulation of fumarate, 2SC and succinated proteins such as succinic-GSH $(24,25)$, marks their potential as biomarkers for the detection of FH-deficient RCCs in tumour tissue and bodily fluids. A combination of these inexpensive, relatively straightforward detection methods that are highly specific to FH-deficiency may by the way forwards for improving detection and diagnosis of this aggressive subtype.

Overall, this study by Gupta and colleagues highlights the potential of IHC to be used as an adjunctive tool in the diagnosis of rare RCC subtypes, which is invaluable given the evidence for these rare subtypes to masquerade behind variant histology. Given that IHC is routinely established in clinical practice to assist the diagnosis of renal tumours (26), it would be relatively straightforward to add SDHA/B and $\mathrm{FH} / 2 \mathrm{SC}$ staining to the IHC panel for clinical use to improve detection of these rare and aggressive subtypes. As highlighted, the ramifications of optimising detection of these subtypes are multi-fold. Detection will affect patients presenting with both localised and advanced disease, it will improve our knowledge of these poorly understood cohorts, and as both RCC subtypes are strongly associated with 
hereditary syndromes, it will have important implications for genetic testing for the patient and their relatives. In particular, diagnosis of aggressive FH-deficient RCC at renal biopsy or resection of a localised tumour will improve stratification of patients into more intensive follow-up regimes (5), whereas in the advanced setting, it will enable stratification of patients into appropriate clinical trials that will ultimately enable data to be gathered for improving systemic and targeted therapies as well as guidelines for these cohorts, which would be in keeping with the climate directed towards subtype-specific management. However, with a very low incidence of SDHand FH-deficient RCCs detected in this and previous similar studies $(13,14,16,22)$, the question to debate is whether or not it would be logical and/or cost-effective to screen the entire RCC cohort to detect a few cases of SDH- or FHdeficient RCC. Perhaps, it is in these cases that physicians and pathologists use their clinical acumen in aligning variant histology with a suspicious clinical history e.g., family or personal history of associated cancer phenotypes, early onset of disease, clinically aggressive phenotype etc, that may really serve in increasing the detection of these tumours within variant RCC subtypes.

\section{Acknowledgments}

Funding: This work was supported by the Wellcome Trust (to C Yong), The Urology Foundation (to C Yong), and by the Medical Research Council (to C Frezza) (MRC_MC_ UU_12022/6).

\section{Footnote}

Conflicts of Interest: C Frezza is adviser of Istesso Limited, and member of the Scientific Advisory Board of Owlstone Medicals. GD Stewart has received educational grants from Pfizer, AstraZeneca and Intuitive Surgical, consultancy fees from Merck, Pfizer, EUSA Pharma and CMR Surgical, travel expenses from Pfizer and speaker fees from Pfizer. C Yong has no conflicts of interest to declare.

Ethical Statement: The authors are accountable for all aspects of the work in ensuring that questions related to the accuracy or integrity of any part of the work are appropriately investigated and resolved.

\section{References}

1. Moch H, Cubilla AL, Humphrey PA, et al. The 2016
WHO Classification of Tumours of the Urinary System and Male Genital Organs_-Part A: Renal, Penile, and Testicular Tumours. Eur Urol 2016;70:93-105.

2. Schmidt LS, Linehan WM. Genetic predisposition to kidney cancer. Semin Oncol 2016;43:566-74.

3. Ricketts CJ, Cubas AAD, Fan H, et al. The Cancer Genome Atlas Comprehensive Molecular Characterization of Renal Cell Carcinoma. Cell Rep 2018;23:313-26.e5.

4. Frezza C, Pollard PJ, Gottlieb E. Inborn and acquired metabolic defects in cancer. J Mol Med (Berl) 2011;89:213-20.

5. Carlo MI, Hakimi AA, Stewart GD, et al. Familial Kidney Cancer: Implications of New Syndromes and Molecular Insights. Eur Urol 2019. [Epub ahead of print].

6. Linehan WM, Spellman PT, Ricketts CJ, et al. Comprehensive Molecular Characterization of Papillary Renal Cell Carcinoma. N Engl J Med 2016;374:135-45.

7. Ljungberg B, Albiges L, Abu-Ghanem Y, et al. European Association of Urology Guidelines on Renal Cell Carcinoma: The 2019 Update. Eur Urol 2019;75:799-810.

8. Ricketts CJ, Shuch B, Vocke CD, et al. Succinate dehydrogenase kidney cancer: an aggressive example of the Warburg effect in cancer. J Urol 2012;188:2063-71.

9. Gill AJ. Succinate dehydrogenase (SDH)-deficient neoplasia. Histopathology 2018;72:106-16.

10. National Comprehensive Cancer Network. NCCN Clinical Practice Guidelines in Oncology: Kidney Cancer (Version 2.2020). Kidney Cancer 2019. Available online: https://www.nccn.org/professionals/physician_gls/default. aspx

11. Escudier B, Porta C, Schmidinger M, et al. Renal cell carcinoma: ESMO Clinical Practice Guidelines for diagnosis, treatment and follow-up. Ann Oncol 2019;30:706-20.

12. Chen YB, Brannon AR, Toubaji A, et al. Hereditary Leiomyomatosis and Renal Cell Carcinoma Syndromeassociated Renal Cancer: Recognition of the Syndrome by Pathologic Features and the Utility of Detecting Aberrant Succination by Immunohistochemistry. Am J Surg Pathol 2014;38:627-37.

13. Miettinen M, Sarlomo-Rikala M, McCue P, et al. Mapping of succinate dehydrogenase losses in 2258 epithelial neoplasms. Appl Immunohistochem Mol Morphol 2014;22:31-6.

14. Trpkov K, Hes O, Agaimy A, et al. Fumarate Hydratasedeficient Renal Cell Carcinoma Is Strongly Correlated With Fumarate Hydratase Mutation and Hereditary Leiomyomatosis and Renal Cell Carcinoma Syndrome. 
Am J Surg Pathol 2016;40:865-75.

15. Bardella C, El-Bahrawy M, Frizzell N, et al. Aberrant succination of proteins in fumarate hydratase-deficient mice and HLRCC patients is a robust biomarker of mutation status. J Pathol 2011;225:4-11.

16. Cornejo KM, Lu M, Yang P, et al. Succinate Dehydrogenase B (SDHB): A New Prognostic Biomarker in Clear Cell Renal Cell Carcinoma. Hum Pathol 2015;46:820-6.

17. Sciacovelli M, Frezza C. Oncometabolites: Unconventional triggers of oncogenic signalling cascades. Free Radic Biol Med 2016;100:175-81.

18. Yong C, Stewart GD, Frezza C. Oncometabolites in renal cancer: Warburg's hypothesis re-examined. Available online: https://www.repository.cam.ac.uk/ handle/1810/294889

19. Alderson NL, Wang Y, Blatnik M, et al. S-(2-Succinyl) cysteine: a novel chemical modification of tissue proteins by a Krebs cycle intermediate. Arch Biochem Biophys 2006;450:1-8.

20. Gupta S, Swanson AA, Chen YB, et al. Incidence of succinate dehydrogenase and fumarate hydratase-deficient renal cell carcinoma based on immunohistochemical screening with SDHA/SDHB and FH/2SC. Hum Pathol

Cite this article as: Yong C, Stewart GD, Frezza C. Immunohistochemistry as a tool for screening rare renal cancers. Ann Transl Med 2019;7(Suppl 8):S314. doi: 10.21037/ atm.2019.10.18
2019;91:114-22.

21. Vanharanta S, Buchta M, McWhinney SR, et al. EarlyOnset Renal Cell Carcinoma as a Novel Extraparaganglial Component of SDHB-Associated Heritable Paraganglioma. Am J Hum Genet 2004;74:153-9.

22. Smith SC, Trpkov K, Chen YB, et al. Tubulocystic Carcinoma of the Kidney With Poorly Differentiated Foci: A Frequent Morphologic Pattern of Fumarate Hydratasedeficient Renal Cell Carcinoma. Am J Surg Pathol 2016;40:1457-72.

23. Zheng L, MacKenzie ED, Karim SA, et al. Reversed argininosuccinate lyase activity in fumarate hydratasedeficient cancer cells. Cancer Metab 2013;1:12.

24. Sciacovelli M, Gonçalves E, Johnson TI, et al. Fumarate is an epigenetic modifier that elicits epithelial-tomesenchymal transition. Nature 2016;537:544-7.

25. Sullivan LB, Martinez-Garcia E, Nguyen H, et al. The Proto-oncometabolite Fumarate Binds Glutathione to Amplify ROS-Dependent Signaling. Mol Cell 2013;51:236-48.

26. Warren AY, Harrison D. WHO/ISUP classification, grading and pathological staging of renal cell carcinoma: standards and controversies. World J Urol 2018;36:1913-26. 